\title{
DETERMINANT TYPE GENERALIZATIONS OF THE HEINZ-KATO THEOREM VIA THE FURUTA INEQUALITY
}

\author{
TAKAYUKI FURUTA
}

(Communicated by Palle E. T. Jorgensen)

Dedicated to Professor Osamu Takenouchi with respect and affection

\begin{abstract}
A capital letter means a bounded linear operator on a complex Hilbert space $H$. By a nice application of the Furuta inequality, we give two kinds of determinant type generalizations (Theorems 1 and 2 in $\S 1$ ) of the famous and well-known Heinz-Kato theorem containing the terms $T,|T|$, and $\left|T^{*}\right|$.
\end{abstract}

\section{INTRODUCTION}

An operator $T$ is said to be positive if $(T x, x) \geq 0$ for all $x \in H$. We recall the following famous Löwner-Heinz theorem [5,8]. If $A \geq B \geq 0$, then $A^{\alpha} \geq B^{\alpha}$ for each $\alpha \in[0,1]$. There are a lot of proofs of this famous theorem, in particular, an elegant proof given in [9].

Also we recall the following famous Heinz-Kato theorem [5, 7]. If $A$ and $B$ are positive operators such that $\|T x\| \leq\|A x\|$ and $\left\|T^{*} y\right\| \leq\|B y\|$ for all $x, y \in H$, then the following inequality holds: $|(T x, y)| \leq\left\|A^{\alpha} x\right\|\left\|B^{1-\alpha} y\right\|$ for any $0 \leq \alpha \leq 1$.

We have the Furuta inequality [2] as some extension of this Löwner-Heinz theorem as follows: If $A \geq B \geq 0$, then for each $r \geq 0$

(i) $\left(B^{r} A^{p} B^{r}\right)^{1 / q} \geq B^{(p+2 r) / q}$ and

(ii) $A^{(p+2 r) / q} \geq\left(A^{r} B^{p} A^{r}\right)^{1 / q}$

hold for each $p$ and $q$ such that $p \geq 0$ and $q \geq 1$ with $(1+2 r) q \geq p+2 r$.

When we put $r=0$ in (i) or (ii) in the Furuta inequality stated above, we have the famous Löwner-Heinz theorem. Alternative proofs of the Furuta inequality are given in [1, 3,6], and an elementary proof is shown in [4].

In this paper, as an application of the Furuta inequality we shall show Theorems 1 and 2, which are two kinds of determinant type inequalities, and these two theorems yield Theorem 3, which is a generalization of the Heinz-Kato theorem. Also we shall show that any one of these generalizations is equivalent to the Furuta inequality.

Received by the editors May 5, 1992.

1991 Mathematics Subject Classification. Primary 47A30; Secondary 47A63.

Key words and phrases. Positive operator, operator inequality, the Furuta inequality.

(C) 1993 American Mathematical Society $0002-9939 / 93 \$ 1.00+\$ .25$ per page 


\section{Determinant type generalizations of the Heinz-Kato theorem}

Put $f=(1+2 r) \alpha$ and $g=(1+2 s) \beta$ for any $r \geq 0, s \geq 0$, and $\alpha, \beta \in$ $[0,1]$. Let the $n \times n$ determinant $G_{n}^{(1)}(r, s, \alpha, \beta)$ be defined by the following formula for $h=(1+2 r) \alpha+(1+2 s) \beta-1 \geq 0$ and $x_{1}, x_{2}, \ldots, x_{n} \in H$ :

$$
\begin{aligned}
& G_{n}^{(1)}(r, s, \alpha, \beta) \\
& \quad=\left|\begin{array}{ccccc}
\left(|T|^{2 f} x_{1}, x_{1}\right) & \left(T|T|^{h} x_{1}, x_{2}\right) & \left(T|T|^{h} x_{1}, x_{3}\right) & \ldots & \left(T|T|^{h} x_{1}, x_{n}\right) \\
\left(|T|^{h} T^{*} x_{2}, x_{1}\right) & \left(\left|T^{*}\right|^{2 g} x_{2}, x_{2}\right) & \left(\left|T^{*}\right|^{2 g} x_{2}, x_{3}\right) & \ldots & \left(\left|T^{*}\right|^{2 g} x_{2}, x_{n}\right) \\
\left(|T|^{h} T^{*} x_{3}, x_{1}\right) & \left(\left|T^{*}\right|{ }^{2 g} x_{3}, x_{2}\right) & \left(\left|T^{*}\right|^{2 g} x_{3}, x_{3}\right) & \ldots & \left(\left|T^{*}\right|^{2 g} x_{3}, x_{n}\right) \\
\ldots & \ldots & \ldots & \ldots & \ldots \\
\left(|T|^{h} T^{*} x_{n}, x_{1}\right) & \left(\left|T^{*}\right|^{2 g} x_{n}, x_{2}\right) & \left(\left|T^{*}\right|^{2 g} x_{n}, x_{3}\right) & \cdots & \left(\left|T^{*}\right|^{2 g} x_{n}, x_{n}\right)
\end{array}\right| .
\end{aligned}
$$

Theorem 1 (Type 1). Let $T$ be an operator on a Hilbert space $H$. If $A, B_{2}$, $B_{3}, \ldots, B_{n}$ are positive operators such that $\|T x\| \leq\|A x\|$ and $\left\|T^{*} y\right\| \leq\left\|B_{j} y\right\|$ for all $x, y \in H$ and $j=2,3, \ldots, n$, then, for each $r \geq 0$ and $s \geq 0$, the following inequality holds for all $x_{1}, x_{2}, \ldots, x_{n} \in H$ :

$$
\begin{aligned}
& \left(|T|^{2(1+2 r) \alpha} x_{1}, x_{1}\right) \prod_{j=2}^{n}\left(\left|T^{*}\right|^{2(1+2 s) \beta} x_{j}, x_{j}\right) \\
& \leq G_{n}^{(1)}(r, s, \alpha, \beta)+\left(\left(|T|^{2 r} A^{2 p}|T|^{2 r}\right)^{(1+2 r) \alpha /(p+2 r)} x_{1}, x_{1}\right) \\
& \quad \times \prod_{j=2}^{n}\left(\left(\left|T^{*}\right|^{2 s} B_{j}^{2 q}\left|T^{*}\right|^{2 s}\right)^{(1+2 s) \beta /(q+2 s)} x_{j}, x_{j}\right)
\end{aligned}
$$

for any $p \geq 1, q \geq 1$, and $\alpha, \beta \in[0,1]$ such that $(1+2 r) \alpha+(1+2 s) \beta \geq 1$.

In the case $\alpha>0$ and $\beta>0$, the equality in (1) holds for some vectors $x_{1}, x_{2}, \ldots, x_{n} \in H$ iff the following $\left(\mathrm{a}_{1}\right),\left(\mathrm{b}_{1}\right)$, and $\left(\mathrm{c}_{1}\right)$ hold together for some vectors $x_{1}, x_{2}, \ldots, x_{n} \in H$ :

(a $\left.a_{1}\right) \quad\left\{|T|^{2(1+2 r) \alpha} x_{1},|T|^{(1+2 r) \alpha+(1+2 s) \beta-1} T^{*} x_{2},|T|^{(1+2 r) \alpha+(1+2 s) \beta-1} T^{*} x_{3}, \ldots\right.$, $\left.|T|^{(1+2 r) \alpha+(1+2 s) \beta-1} T^{*} x_{n}\right\}$ is a sequence of linearly dependent vectors,

(b 1$) \quad|T|^{2(1+2 r) \alpha} x_{1}=\left(|T|^{2 r} A^{2 p}|T|^{2 r}\right)^{(1+2 r) \alpha /(p+2 r)} x_{1}$,

(c) $\left|T^{*}\right|^{2(1+2 s) \beta} x_{j}=\left(\left|T^{*}\right|^{2 s} B_{J}^{2 q}\left|T^{*}\right|^{2 S}\right)^{(1+2 s) \beta /(q+2 s)} x_{j}$ for $j=2,3, \ldots, n$.

We define $f, g$, and $h$ the same as in the definition of $G_{n}^{(1)}(r, s, \alpha, \beta)$; that is, $f=(1+2 r) \alpha$ and $g=(1+2 s) \beta$ for any $r \geq 0, s \geq 0$, and $\alpha, \beta \in[0,1]$.

Let the $2 n \times 2 n$ determinant $G_{2 n}^{(2)}(r, s, \alpha, \beta)$ be defined by the following formula for $h=(1+2 r) \alpha+(1+2 s) \beta-1 \geq 0$ and $x_{1}, x_{2}, \ldots, x_{2 n-1}, x_{2 n} \in H$ :

$$
\begin{aligned}
& G_{2 n}^{(2)}(r, s, \alpha, \beta) \\
& \quad=\left|\begin{array}{ccccc}
\left(|T|^{2} x_{1}, x_{1}\right) & \left(T|T|^{h} x_{1}, x_{2}\right) & \ldots & \left(|T|^{2 f} x_{1}, x_{2 n-1}\right) & \left(T|T|^{h} x_{1}, x_{2 n}\right) \\
\left(|T|^{h} T^{*} x_{2}, x_{1}\right) & \left(\left|T^{*}\right|^{2} x_{2}, x_{2}\right) & \cdots & \left(|T|^{h} T^{*} x_{2}, x_{2 n-1}\right) & \left(\left|T^{*}\right|^{2} x_{2}, x_{2 n}\right) \\
\cdots & \cdots & \cdots & \ldots & \ldots \\
\left(|T|^{2 f} x_{2 n-1}, x_{1}\right) & \left(T|T|^{h} x_{2 n-1}, x_{2}\right) & \cdots & \left(|T|^{2 f} x_{2 n-1}, x_{2 n-1}\right) & \left(T|T|^{h} x_{2 n-1}, x_{2 n}\right) \\
\left(|T|^{h} T^{*} x_{2 n}, x_{1}\right) & \left(\left|T^{*}\right|^{2 g} x_{2 n}, x_{2}\right) & \cdots & \left(|T|^{h} T^{*} x_{2 n}, x_{2 n-1}\right) & \left(\left|T^{*}\right|^{28} x_{2 n}, x_{2 n}\right)
\end{array}\right|
\end{aligned}
$$

Theorem 2 (Type 2). Let $T$ be an operator on a Hilbert space $H$. If $A_{1}$, $A_{3}, \ldots, A_{2 n-1}$ and $B_{2}, B_{4}, \ldots, B_{2 n}$ are positive operators such that $\|T x\| \leq$ $\left\|A_{2 j-1} x\right\|$ and $\left\|T^{*} y\right\| \leq\left\|B_{2 j} y\right\|$ for all $x, y \in H$ and $j=1,2, \ldots, n$, then, for each $r \geq 0$ and $s \geq 0$, the following inequality holds for all $x_{1}, x_{2}, \ldots, x_{2 n-1}$, 
$x_{2 n} \in H$ :

$$
\begin{aligned}
& \prod_{j=1}^{n}\left\{\left(|T|^{2(1+2 r) \alpha} x_{2 j-1}, x_{2 j-1}\right)\left(\left|T^{*}\right|^{2(1+2 s) \beta} x_{2 i}, x_{2 j}\right)\right\} \\
& \leq G_{2 n}^{(2)}(r, s, \alpha, \beta)+\prod_{j=1}^{n}\left\{\left(\left(|T|^{2 r} A_{2 j-1}^{2 p}|T|^{2 r}\right)^{(i+2 r) \alpha /(p+2 r)} x_{2 j-1}, x_{2 j-1}\right)\right. \\
& \left.\quad\left(\left(\left|T^{*}\right|^{2 s} B_{j}^{2 q}\left|T^{*}\right|^{2 s}\right)^{(1+2 s) \beta /(q+2 s)} x_{2 j}, x_{2 j}\right)\right\}
\end{aligned}
$$

for any $p \geq 1, q \geq 1$, and $\alpha, \beta \in[0,1]$ such that $(1+2 r) \alpha+(1+2 s) \beta \geq 1$.

In the case $\alpha>0$ and $\beta>0$, the equality in (2) holds for some vertors $x_{1}, x_{2}, \ldots, x_{2 n-1}, x_{2 n} \in H$ iff the following $\left(\mathrm{a}_{2}\right),\left(\mathrm{b}_{2}\right)$, and $\left(\mathrm{c}_{2}\right)$ hold together for some vectors $x_{1}, x_{2}, \ldots, x_{2 n-1}, x_{2 n} \in H$ :

$\left(\mathrm{a}_{2}\right)$

$$
\begin{aligned}
& \left\{|T|^{2(1+2 r) \alpha} x_{1},|T|^{(1+2 r) \alpha+(1+2 s) \beta-1} T^{*} x_{2},|T|^{2(1+2 r) \alpha} x_{3},\right. \\
& \left.\quad|T|^{(1+2 r) \alpha+(1+2 s) \beta-1} T^{*} x_{4}, \ldots,|T|^{2(1+2 r) \alpha} x_{2 n-1},|T|^{(1+2 r) \alpha+(1+2 s) \beta-1} T^{*} x_{2 n}\right\}
\end{aligned}
$$

is a sequence of linearly dependent vectors,

(b $) \quad|T|^{2(1+2 r) \alpha} x_{2 j-1}=\left(|T|^{2 r} A_{2 j-1}^{2 p}|T|^{2 r}\right)^{(1+2 r) \alpha /(p+2 r)} x_{2 j-1}$ for $j=1,2, \ldots$, $n$,

(c) $\left|T^{*}\right|^{2(1+2 s) \beta} x_{2 j}=\left(\left|T^{*}\right|^{2 s} B_{2 j}^{2 q}\left|T^{*}\right|^{2 s}\right)^{(1+2 s) \beta /(q+2 s)} x_{2 j}$ for $j=1,2, \ldots, n$.

Theorem 3. Let $T$ be an operator on a Hilbert space $H$. If $A$ and $B$ are positive operators such that $\|T x\| \leq\|A x\|$ and $\left\|T^{*} y\right\| \leq\|B y\|$ for all $x, y \in H$, then, for each $r \geq 0$ and $s \geq 0$, the following inequality holds for all $x, y \in H$ :

(3)

$$
\begin{aligned}
& \left|\left(T|T|^{(1+2 r) \alpha+(1+2 s) \beta-1} x, y\right)\right|^{2} \\
& \quad \leq\left(\left(|T|^{2 r} A^{2 p}|T|^{2 r}\right)^{(1+2 r) \alpha /(p+2 r)} x, x\right)\left(\left(\left|T^{*}\right|^{2 s} B^{2 q}\left|T^{*}\right|^{2 s}\right)^{(1+2 s) \beta /(q+2 s)} y, y\right)
\end{aligned}
$$

for any $p \geq 1, q \geq 1$, and $\alpha, \beta \in[0,1]$ such that $(1+2 r) \alpha+(1+2 s) \beta \geq 1$.

In the case $\alpha>0$ and $\beta>0$, the equality in (3) holds for some vectors $x$ and $y \in H$ iff the following $\left(\mathrm{a}_{3}\right),\left(\mathrm{b}_{3}\right)$, and $\left(\mathrm{c}_{3}\right)$ hold together for some $x$ and $y \in H$ :

(a) $|T|^{2(1+2 r) \alpha} x$ and $|T|^{(1+2 r) \alpha+(1+2 s) \beta-1} T^{*} y$ are linearly dependent,

(b) $\quad|T|^{2(1+2 r) \alpha} x=\left(|T|^{2 r} A^{2 p}|T|^{2 r}\right)^{(1+2 r) \alpha /(p+2 r)} x$,

(c) $\left|T^{*}\right|^{2(1+2 s) \beta} y=\left(\left|T^{*}\right|^{2 s} B^{2 q}\left|T^{*}\right|^{2 s}\right)^{(1+2 s) \beta /(q+2 s)} y$.

Remark 1 . We remark that the condition $(1+2 r) \alpha+(1+2 s) \beta \geq 1$ in Theorems 1,2 , and 3 is unnecessary if $T$ is a positive operator or invertible operator. This is easily seen in the proofs of their results.

Theorem 4. Let $T$ be an operator on a Hilbert space $H$. If $A$ and $B$ are positive operators such that $\|T x\| \leq\|A x\|$ and $\left\|T^{*} y\right\| \leq\|B y\|$ for all $x, y \in H$, then the following inequality holds for all $x, y \in H$ :

$$
\left|\left(T|T|^{\alpha+\beta-1} x, y\right)\right| \leq\left\|A_{x}^{\alpha}\right\|\left\|B^{\beta} y\right\|
$$

for any $\alpha$ and $\beta$ such that $\alpha, \beta \in[0,1]$ and $\alpha+\beta \geq 1$. 
In the case $\alpha>0$ and $\beta>0$, the equality in (4) holds for some $x$ and $y$ iff $|T|^{2 \alpha} x$ and $|T|^{\alpha+\beta-1} T^{*} y$ are linearly dependent and $|T|^{2 \alpha} x=A^{2 \alpha} x$ and $\left|T^{*}\right|^{2 \beta} y=B^{2 \beta} y$ hold for some $x$ and $y$ together.

Theorem A (Heinz-Kato). Let $T$ be an operator on a Hilbert space $H$. If $A$ and $B$ are positive operators such that $\|T x\| \leq\|A x\|$ and $\left\|T^{*} y\right\| \leq\|B y\|$ for all $x, y \in H$, then the following inequality holds for all $x, y \in H$ :

$$
|(T x, y)| \leq\left\|A^{\alpha} x\right\|\left\|B^{1-\alpha} y\right\| \quad \text { for any } \alpha \in[0,1] .
$$

\section{Proofs of the Results}

At first we cite the following well-known folk lemma.

Lemma. For any vectors $x_{1}, x_{2}, \ldots, x_{n} \in H$, let $G_{n}$ be the determinant of the square matrix of order $n$ defined by $G_{n}=\left|\left(\left(x_{j}, x_{k}\right)\right)\right|$. Then $0 \leq G_{n}$. The equality holds if and only if $x_{1}, x_{2}, \ldots, x_{n}$ are linearly dependent.

In order to give proofs of the results, we need the following inequalities which are equivalent to the Furuta inequality [2].

Theorem B. If $A \geq B \geq 0$, then for each $r \geq 0$

$$
\left(B^{r} A^{p} B^{r}\right)^{(1+2 r) \theta /(p+2 r)} \geq B^{(1+2 r) \theta}
$$

and

$$
A^{(1+2 r) \theta} \geq\left(A^{r} B^{p} A^{r}\right)^{(1+2 r) \theta /(p+2 r)}
$$

hold for any $p \geq 1$ and $\theta \in[0,1]$.

Proof of Theorem 1. Let $N(X)$ denote the kernel of an operator $X$. Let $T=$ $U|T|$ be the polar decomposition of an operator $T$, where $|T|=\left(T^{*} T\right)^{1 / 2}$ and $U$ is a partial isometry operator with $N(U)=N(|T|)$.

The case $g=(1+2 s) \beta>0$. In the Lemma we replace $x_{1}$ by $|T|^{f} x_{1}$ and $x_{k}$ by $|T|^{g} U^{*} x_{k}$ for $k=2,3, \ldots, n$. Then we have

$$
\begin{aligned}
\left(|T|^{f} x_{1},|T|^{g} U^{*} x_{k}\right) & =\left(U|T|^{f+g} x_{1}, x_{k}\right) \\
& =\left(T|T|^{f+g-1} x_{1}, x_{k}\right) \text { for } k=2,3, \ldots, n
\end{aligned}
$$

and

$$
\begin{aligned}
\left(|T|^{g} U^{*} x_{j},|T|^{g} U^{*} x_{k}\right) & =\left(U|T|^{2 g} U^{*} x_{j}, x_{k}\right) \\
& =\left(\left|T^{*}\right|^{2 g} x_{j}, x_{k}\right) \text { for } j, k=2,3, \ldots, n,
\end{aligned}
$$

since $\left|T^{*}\right|^{2 g}=U|T|^{2 g} U^{*}$ holds for any positive number $g$ in general.

Thus we can construct $G_{n}^{(1)}(r, s, \alpha, \beta)$ defined in $\S 1$. By the Lemma we have

$$
0 \leq G_{n}^{(1)}(r, s, \alpha, \beta) \text {. }
$$

The two hypotheses $\|T x\| \leq\|A x\|$ and $\left\|T^{*} y\right\| \leq\left\|B_{j} y\right\|$ for all $x, y \in H$ and $j=2,3, \ldots, n$ are equivalent to the following (8) and (9) respectively:

$$
|T|^{2} \leq A^{2}
$$

and

$$
\left|T^{*}\right|^{2} \leq B_{j}^{2} \quad \text { for } j=2,3, \ldots, n \text {. }
$$


Applying Theorem $\mathrm{B}(\mathrm{i})$ to (8), for each $r \geq 0$ we have

$$
\left(|T|^{2(1+2 r) \alpha} x_{1}, x_{1}\right) \leq\left(\left(|T|^{2 r} A^{2 p}|T|^{2 r}\right)^{(1+2 r) \alpha /(p+2 r)} x_{1}, x_{1}\right)
$$

for any $p \geq 1$ and $\alpha \in[0,1]$.

Also applying Theorem $\mathrm{B}(\mathrm{i})$ to (9), for each $s \geq 0$ we have

$$
\left(\left|T^{*}\right|^{2(1+2 s) \beta} x_{j}, x_{j}\right) \leq\left(\left(\left|T^{*}\right|^{2 s} B_{j}^{2 q}\left|T^{*}\right|^{2 s}\right)^{(1+2 s) \beta /(q+2 s)} x_{j}, x_{j}\right)
$$

for any $q \geq 1, \beta \in[0,1]$, and $j=2, \ldots, n$. Hence the following inequality (1) holds by (7), (10), and (11); that is, for each $r \geq 0$ and $s \geq 0$ and all $x_{1}, x_{2}, \ldots, x_{n} \in H$

$$
\begin{aligned}
\left(|T|^{2(1+2 r) \alpha} x_{1}, x_{1}\right) \prod_{j=2}^{n}\left(\left|T^{*}\right|^{2(1+2 s) \beta} x_{j}, x_{j}\right) \\
\leq G_{n}^{(1)}(r, s, \alpha, \beta)+\left(\left(|T|^{2 r} A^{2 p}|T|^{2 r}\right)^{(1+2 r) \alpha /(p+2 r)} x_{1}, x_{1}\right) \\
\quad \times \prod_{j=2}^{n}\left(\left(\left|T^{*}\right|^{2 s} B_{j}^{2 q}\left|T^{*}\right|^{2 s}\right)^{(1+2 s) \beta /(q+2 s)} x_{j}, x_{j}\right)
\end{aligned}
$$

for any $p \geq 1, q \geq 1$, and $\alpha, \beta \in[0,1]$ such that $(1+2 r) \alpha+(1+2 s) \beta \geq 1$.

The case $g=(1+2 s) \beta=0$. The result is obvious, so we omit its description.

We cite the following obvious results to scrutinize the equality case in (1):

$$
\begin{aligned}
& \text { For a positive operator } S,(S x, x)=0 \text { holds for some vector } \\
& x \text { iff } S x=0 \text { holds for some vector } x .
\end{aligned}
$$

$$
\text { For a positive operator } S, N\left(S^{q}\right)=N(S) \text { holds for any positive }
$$
number $q$.

In the case $f=(1+2 r) \alpha>0$ and $g=(1+2 s) \beta>0$, the equality in (7) holds iff

$$
\left\{|T|^{f} x_{1},|T|^{g} U^{*} x_{2},|T|^{g} U^{*} x_{3}, \ldots,|T|^{g} U^{*} x_{n}\right\}
$$

is a sequence of linearly dependent vectors. That is,

$$
\left\{|T|^{2 f} x_{1},|T|^{f+g-1}|T| U^{*} x_{2},|T|^{f+g-1}|T| U^{*} x_{3}, \ldots,|T|^{f+g-1}|T| U^{*} x_{n}\right\}
$$

is a sequence of linearly dependent vectors by $(* *)$; namely,

$$
\left\{|T|^{2 f} x_{1},|T|^{f+g-1} T^{*} x_{2},|T|^{f+g-1} T^{*} x_{3}, \ldots,|T|^{f+g-1} T^{*} x_{n}\right\}
$$

is a sequence of linearly dependent vectors. That is, $(12)$ is $\left(a_{1}\right)$ in Theorem 1.

The equality in (10) holds for some vector $x_{1}$ iff

$$
\left(|T|^{2 r} A^{2 p}|T|^{2 r}\right)^{(1+2 r) \alpha /(p+2 r)} x_{1}=|T|^{2(1+2 r) \alpha} x_{1}
$$

holds for some vector $x_{1}$ by $(*)$, and also the equality in (11) holds for some vectors $x_{j}$ for $j=1,2, \ldots, n$, iff

$$
\left(\left|T^{*}\right|^{2 s} B_{j}^{2 q}\left|T^{*}\right|^{2 s}\right)^{(1+2 s) \beta /(q+2 s)} x_{j}=\left|T^{*}\right|^{2(1+2 s) \beta} x_{j}
$$

holds for some vectors $x_{j}$ for $j=1,2, \ldots, n$ by $(*)$.

The case $\alpha>0$ and $\beta>0$ is equivalent to the case $(1+2 r) \alpha>0$ and $(1+2 s) \beta>0$. In this case, the equality in (1) holds iff the equalities in (7), $(10)$, and (11) hold together; that is, the conditions $\left(a_{1}\right),\left(b_{1}\right)$, and $\left(c_{1}\right)$ in 
Theorem 1 hold together by (12)-(14). Therefore, the proof of the equality in (1) is complete.

Hence, the proof of Theorem 1 is complete.

Proof of Theorem 2. The case $g=(1+2 s) \beta>0$. In the Lemma we replace $x_{2 j}$ by $|T|^{g} U^{*} x_{2 j}$ for $j=1,2, \ldots, n$ and also $x_{2 j-1}$ by $|T|^{f} x_{2 j-1}$ for $j=$ $1,2, \ldots, n$. Then we have

$$
\begin{aligned}
& \left(|T|^{f} x_{2 j-1},|T|^{g} U^{*} x_{2 k}\right)=\left(U|T|^{f+g} x_{2 j-1}, x_{2 k}\right) \\
& \quad=\left(T|T|^{f+g-1} x_{2 j-1}, x_{2 k}\right) \text { for } j, k=1,2, \ldots, n
\end{aligned}
$$

and

$$
\begin{aligned}
\left(|T|^{g} U^{*} x_{2 j},|T|^{g} U^{*} x_{2 k}\right) & =\left(U|T|^{2 g} U^{*} x_{2 j}, x_{2 k}\right) \\
& =\left(\left|T^{*}\right|^{2 g} x_{2 j}, x_{2 k}\right) \text { for } j, k=1,2, \ldots, n,
\end{aligned}
$$

since $\left|T^{*}\right|^{2 g}=U|T|^{2 g} U^{*}$ holds for any positive number $g$ in general. have

Thus we can construct $G_{2 n}^{(2)}(r, s, \alpha, \beta)$ defined in $\S 1$. By the Lemma, we

$$
0 \leq G_{2 n}^{(2)}(r, s, \alpha, \beta) .
$$

The two hypotheses $\|T x\| \leq\left\|A_{2 j-1} x\right\|$ and $\left\|T^{*} y\right\| \leq\left\|B_{2 j} y\right\|$ for all $x, y \in$ $H$ and $j=1,2, \ldots, n$ are equivalent respectively to

$$
|T|^{2} \leq A_{2 j-1}^{2} \quad \text { for } j=1,2, \ldots, n
$$

and

$$
\left|T^{*}\right|^{2} \leq B_{2 j}^{2} \quad \text { for } j=1,2, \ldots, n .
$$

Applying Theorem $\mathrm{B}(\mathrm{i})$ to (18), for each $r \geq 0$ we have

$$
\left(|T|^{2(1+2 r) \alpha} x_{2 j-1}, x_{2 j-1}\right) \leq\left(\left(|T|^{2 r} A_{2 j-1}^{2 p}|T|^{2 r}\right)^{(1+2 r) \alpha /(p+2 r)} x_{2 j-1}, x_{2 j-1}\right)
$$

for any $p \geq 1, \alpha \in[0,1]$, and $j=1,2, \ldots, n$. Also applying Theorem $\mathrm{B}(\mathrm{i})$ to (19), for each $s \geq 0$ we have

$$
\left(\left|T^{*}\right|^{2(1+2 s) \beta} x_{2 j}, x_{2 j}\right) \leq\left(\left(\left|T^{*}\right|^{2 s} B_{j}^{2 q}\left|T^{*}\right|^{2 s}\right)^{(1+2 s) \beta /(q+2 s)} x_{2 j}, x_{2 j}\right)
$$

for any $q \geq 1, \beta \in[0,1]$, and $j=1,2, \ldots, n$. Hence the following inequality (2) holds by (17), (20), and (21); that is, for each $r \geq 0, s \geq 0$, and for all $x_{1}, x_{2}, \ldots, x_{2 n-1}, x_{2 n} \in H$ :

$$
\prod_{j=1}^{n}\left\{\left(|T|^{2(1+2 r) \alpha} x_{2 j-1}, x_{2 j-1}\right)\left(\left|T^{*}\right|^{2(1+2 s) \beta} x_{2 j}, x_{2 j}\right)\right\}
$$

$$
\begin{array}{r}
\leq G_{2 n}^{(2)}(r, s, \alpha, \beta)+\prod_{j=1}^{n}\left\{\left(\left(|T|^{2 r} A_{2 j-1}^{2 p}|T|^{2 r}\right)^{(1+2 r) \alpha /(p+2 r)} x_{2 j-1}, x_{2 j-1}\right)\right. \\
\left.\left(\left(\left|T^{*}\right|^{2 s} B_{j}^{2 q}\left|T^{*}\right|^{2 s}\right)^{(1+2 s) \beta /(q+2 s)} x_{2 j}, x_{2 j}\right)\right\}
\end{array}
$$

for any $p \geq 1, q \geq 1$, and $\alpha, \beta \in[0,1]$ such that $(1+2 r) \alpha+(1+2 s) \beta \geq 1$.

The case $g=(1+2 s) \beta=0$. The result is obvious, so we omit its description.

Next we shall scrutinize the equality case in (2). In the case $f=(1+2 r) \alpha>0$ and $g=(1+2 s) \beta>0$, the equality in (17) holds iff

$$
\left\{|T|^{f} x_{1},|T|^{g} U^{*} x_{2},|T|^{f} x_{3},|T|^{g} U^{*} x_{4}, \ldots,|T|^{f} x_{2 n-1},|T|^{g} U^{*} x_{n}\right\}
$$


is a sequence of linearly dependent vectors by the Lemma. That is,

$$
\begin{aligned}
\left\{|T|^{2 f} x_{1},|T|^{f+g-1}|T| U^{*} x_{2},|T|^{2 f} x_{3},\right. & |T|^{f+g-1}|T| U^{*} x_{4}, \ldots, \\
& \left.|T|^{2 f} x_{2 n-1},|T|^{f+g-1}|T| U^{*} x_{2 n}\right\}
\end{aligned}
$$

is a sequence of linearly dependent vectors by $(* *)$; namely,

$$
\begin{aligned}
\left\{|T|^{2 f} x_{1},|T|^{f+g-1} T^{*} x_{2},|T|^{2 f} x_{3},\right. & |T|^{f+g-1} T^{*} x_{4}, \ldots, \\
& \left.|T|^{2 f} x_{2 n-1},|T|^{f+g-1} T^{*} x_{2 n}\right\}
\end{aligned}
$$

is a sequence of linearly dependent vectors. That is, $(22)$ is $\left(a_{2}\right)$ in Theorem 2.

The equality in (20) holds for some vectors $x_{2 j-1}$ iff

$$
\left(|T|^{2 r} A^{2 p}|T|^{2 r}\right)^{(1+2 r) \alpha /(p+2 r)} x_{2 j-1}=|T|^{2(1+2 r) \alpha} x_{2 j-1}
$$

holds for some vectors $x_{2 j-1}$ for $j=1,2, \ldots, n$ by $(*)$, and also the equality in (21) holds for some vectors $x_{2 j}$ iff

$$
\left(\left|T^{*}\right|^{2 s} B_{j}^{2 q}\left|T^{*}\right|^{2 s}\right)^{(1+2 s) \beta /(q+2 s)} x_{2 j}=\left|T^{*}\right|^{2(1+2 s) \beta} x_{2 j}
$$

holds for some vectors $x_{2 j}$ for $j=1,2, \ldots, n$ by $(*)$.

The case $\alpha>0$ and $\beta>0$ is equivalent to the case $(1+2 r) \alpha>0$ and $(1+2 s) \beta>0$. In this case, the equality in (2) holds iff the equalities in (17), (20), and (21) hold together; that is, the conditions $\left(a_{2}\right),\left(b_{2}\right)$, and $\left(c_{2}\right)$ in Theorem 2 hold together by (22)-(24). Hence the proof of Theorem 2 is complete, together with the proof of the equality in (2).

Remark 2. Equivalent conditions. In the special case $\alpha=0$, scrutiny of the equality in (1) is obvious. In the case $\beta=0$, scrutiny of the equality in (1) is also obvious. In the case $\alpha>0$ and $\beta>0$ in Theorem 1 , we shall show that the following two conditions $\left(a_{1}\right)$ in Theorem 1 and $\left(a_{1}^{\prime}\right)$ are equivalent:

(a $\left.a_{1}\right) \quad\left\{|T|^{2(1+2 r) \alpha} x_{1},|T|^{(1+2 r) \alpha+(1+2 s) \beta-1} T^{*} x_{2},|T|^{(1+2 r) \alpha+(1+2 s) \beta-1} T^{*} x_{3}, \ldots\right.$, $\left.|T|^{(1+2 r) \alpha+(1+2 s) \beta-1} T^{*} x_{n}\right\}$ is a sequence of linearly dependent vectors.

$\left(\mathrm{a}_{1}^{\prime}\right) \quad\left\{T|T|^{(1+2 r) \alpha+(1+2 s) \beta-1} x_{1},\left|T^{*}\right|^{2(1+2 s) \beta} x_{2},\left|T^{*}\right|^{2(1+2 s) \beta} x_{3}, \ldots\right.$, $\left.\left|T^{*}\right|^{2(1+2 s) \beta} x_{n}\right\}$ is a sequence of linearly dependent vectors.

In fact the former condition $\left(a_{1}\right)$ is equivalent to

$$
\left\{|T|^{f} x_{1},|T|^{g} U^{*} x_{2},|T|^{g} U^{*} x_{3}, \ldots,|T|^{g} U^{*} x_{n}\right\}
$$

is a sequence of linearly dependent vectors, as is easily seen in the proof of the equality in (7), and this condition is equivalent to

$$
\left\{U|T|^{f+g} x_{1}, U|T|^{2 g} U^{*} x_{2}, U|T|^{2 g} U^{*} x_{3}, \ldots, U|T|^{2 g} U^{*} x_{n}\right\}
$$

is a sequence of linearly dependent vectors by $(* *)$ and $N(|T|)=N(U)$. That is,

$$
\left\{T|T|^{f+g-1} x_{1},\left|T^{*}\right|^{2 g} x_{2},\left|T^{*}\right|^{2 g} x_{3}, \ldots,\left|T^{*}\right|^{2 g} x_{n}\right\}
$$

is a sequence of linearly dependent vectors; that is, $\left(\mathrm{a}_{1}^{\prime}\right)$ holds.

In the case $\alpha>0$ and $\beta>0$, we shall show that the following $\left(a_{2}\right)$ and $\left(\mathbf{a}_{2}^{\prime}\right)$ are equivalent:

$\left(\mathrm{a}_{2}\right) \quad\left\{|T|^{2(1+2 r) \alpha} x_{1},|T|^{(1+2 r) \alpha+(1+2 s) \beta-1} T^{*} x_{2},|T|^{2(1+2 r) \alpha} x_{3}\right.$, $\left.|T|^{2(1+2 r) \alpha+(1+2 s) \beta-1} T^{*} x_{4}, \ldots,|T|^{2(1+2 r) \alpha} x_{2 j-1},|T|^{(1+2 r) \alpha+(1+2 s) \beta-1} T^{*} x_{2 n}\right\}$ is a sequence of linearly dependent vectors. 
$\left(\mathrm{a}_{2}^{\prime}\right) \quad\left\{T|T|^{(1+2 r) \alpha+(1+2 s) \beta-1} x_{1},\left|T^{*}\right|^{2(1+2 s) \beta} x_{2}, T|T|^{(1+2 r) \alpha+(1+2 s) \beta-1} x_{3}\right.$, $\left.\left|T^{*}\right|^{2(1+2 s) \beta} x_{4}, \ldots, T|T|^{(1+2 r) \alpha+(1+2 s) \beta-1} x_{2 n-1},\left|T^{*}\right|^{2(1+2 s) \beta} x_{2 n}\right\}$ is a sequence of linearly dependent vectors.

In fact the former condition $\left(a_{2}\right)$ is equivalent to

$$
\left\{|T|^{f} x_{1},|T|^{g} U^{*} x_{2},|T|^{f} x_{3},|T|^{g} U^{*} x_{4}, \ldots,|T|^{f} x_{2 n-1},|T|^{g} U^{*} x_{2 n}\right\}
$$

is a sequence of linear dependent vectors, as is easily seen in the proof of the equality in (17), and this condition is equivalent to

$$
\begin{aligned}
\left\{U|T|^{f+g} x_{1}, U|T|^{2 g} U^{*} x_{2}, U|T|^{f+g} x_{3}, U|T|^{2 g} U^{*} x_{4}, \ldots,\right. & \left.U|T|^{f+g} x_{2 n-1}, U|T|^{2 g} U^{*} x_{2 n}\right\}
\end{aligned}
$$

is a sequence of linearly dependent vectors by $(* *)$ and $N(|T|)=N(U)$. That is,

$$
\begin{aligned}
\left\{T|T|^{f+g-1} x_{1},\left|T^{*}\right|^{2 g} x_{2}, T|T|^{f+g-1} x_{3},\left|T^{*}\right|^{2 g} x_{4}, \ldots,\right. \\
\left.T|T|^{f+g-1} x_{2 n-1},\left|T^{*}\right|^{2 g} x_{2 n}\right\}
\end{aligned}
$$

is a sequence of linearly dependent vectors; that is, $\left(a_{2}^{\prime}\right)$ holds.

Proof of Theorem 3. In Theorem 1, we put $n=2$. Then for each $r \geq 0$ and $s \geq 0$ the following inequality holds for all $x, y \in H$ by Theorem 1:

$$
\begin{aligned}
&\left(|T|^{(1+2 r) \alpha} x, x\right)\left(\left|T^{*}\right|^{2(1+2 s) \beta} y, y\right) \\
& \leq G_{2}^{(1)}(r, s, \alpha, \beta)+\left(\left(|T|^{2 r} A^{2 p}|T|^{2 r}\right)^{(1+2 r) \alpha /(p+2 r)} x, x\right) \\
& \quad \times\left(\left(\left|T^{*}\right|^{2 s} B^{2 q}\left|T^{*}\right|^{2 s}\right)^{(1+2 s) \beta /(q+2 s)} y, y\right) .
\end{aligned}
$$

That is,

$$
\begin{aligned}
& \left(|T|^{(1+2 r) \alpha} x, x\right)\left(\left|T^{*}\right|^{2(1+2 s) \beta} y, y\right) \\
& \quad \leq\left(|T|^{(1+2 r) \alpha} x, x\right)\left(\left|T^{*}\right|^{2(1+2 s) \beta} y, y\right)-\left|\left(T|T|^{2(1+2 r) \alpha+2(1+2 s) \beta-1} x, y\right)\right|^{2} \\
& \quad+\left(\left(|T|^{2 r} A^{2 p}|T|^{2 r}\right)^{(1+2 r) \alpha /(p+2 r)} x, x\right)\left(\left(\left|T^{*}\right|^{2 s} B^{2 q}\left|T^{*}\right|^{2 s}\right)^{(1+2 s) \beta /(q+2 s)} y, y\right) ;
\end{aligned}
$$

namely,

$$
\begin{aligned}
&\left|\left(T|T|^{(1+2 r) \alpha+(1+2 s) \beta-1} x, y\right)\right|^{2} \\
& \leq\left(\left(|T|^{2 r} A^{2 p}|T|^{2 r}\right)^{(1+2 r) \alpha /(p+2 r)} x, x\right) \\
& \times\left(\left(\left|T^{*}\right|^{2 s} B^{2 q}\left|T^{*}\right|^{2 s}\right)^{(1+2 s) \beta /(q+2 s)} y, y\right)
\end{aligned}
$$

for any $y \geq 1, q \geq 1$, and $\alpha, \beta \in[0,1]$ such that $(1+2 r) \alpha+(1+2 s) \beta \geq 1$. Hence the proof of Theorem 3 is complete.

Also, Theorem 3 easily follows by Theorem 2, putting $n=1$.

Proof of Theorem 4. Thecrem 4 easily follows by Theorem 3, putting $r=s=0$.

Proof of Theorem A. Theorem A easily follows by Theorem 4, putting $\alpha+\beta=1$.

\section{THE EQUiVALENCE RELATION BETWEeN}

\section{THEOREMS 1, 2, AND B}

Theorems 1 and 2 are proved by Theorem $\mathrm{B}$, which is an extension of the Löwner-Heinz theorem. In this section, conversely we shall show that Theorem $\mathrm{B}$ can be derived from Theorems 1 or 2 as follows: 
Theorem $1 \Rightarrow$ Theorem B. In (1) of Theorem 1, we put $n=2, T=B$, $\alpha=\beta, r=s$, and also we put $x_{1}=x_{2}=x$. Then the hypothesis $\|T x\| \leq\|A x\|$ is equivalent to $B^{2} \leq A^{2}$. Theorem 1 and Remark 1 ensure the following inequality for each $r \geq 0$ and $\alpha \in[0,1]$ :

$$
\begin{aligned}
\left|\left(B^{2(1+2 r) \alpha} x, x\right)\right|^{2} \leq & G_{2}^{(1)}(r, r, \alpha, \alpha) \\
& +\left(\left(B^{2 r} A^{2 p} B^{2 r}\right)^{(1+2 r) \alpha /(p+2 r)} x, x\right)\left(B^{2(1+2 r) \alpha} x, x\right)
\end{aligned}
$$

for any $p \geq 1$. In this case

$$
G_{2}^{(1)}(r, r, \alpha, \alpha)=\left|\left(B^{2(1+2 r) \alpha} x, x\right)\right|^{2}-\left|\left(B^{2(1+2 r) \alpha} x, x\right)\right|^{2}=0,
$$

so we have for each $r \geq 0$ and $\alpha \in[0,1]$

$$
\left|\left(B^{2(1+2 r) \alpha} x, x\right)\right| \leq\left(\left(B^{2 r} A^{2 p} B^{2 r}\right)^{(1+2 r) \alpha /(p+2 r)} x, x\right) \text { for any } p \geq 1 .
$$

Then by (25) we have for each $r \geq 0$

$$
B^{2(1+2 r) \alpha} \leq\left(B^{2 r} A^{2 p} B^{2 r}\right)^{(1+2 r) \alpha /(p+2 r)} \text { for any } p \geq 1
$$

holds under the hypothesis $B^{2} \leq A^{2}$, and the inequality (26) is equivalent to Theorem $B(i)$, which is also equivalent to Theorem $B(i i)$.

Theorem $2 \Rightarrow$ Theorem B. Also putting $n=1$ in Theorem 2, we can show that Theorem B can be derived from Theorem 2 by the same way as in the proof that Theorem $1 \Rightarrow$ Theorem B.

Hence Theorems 1, 2, and B are mutually equivalent.

\section{REFERENCES}

1. M. Fujii, Furuta's inequality and its mean theoretic approach, J. Operator Theory 23 (1990), 67-72.

2. T. Furuta, $A \geq B \geq 0$ assures $\left(B^{r} A^{p} B^{r}\right)^{1 / q} \geq B^{(p+2 r) / q}$ for $r \geq 0, p \geq 0, q \geq 1$ with $(1+2 r) q \geq p+2 r$, Proc. Amer. Math. Soc. 101 (1987), 85-88.

3. $\ldots$ A proof via operator means of an order preserving inequality, Linear Algebra Appl. 113 (1989), 129-130.

4. $\frac{}{126}$, Elementary proof of an order preserving inequality, Proc. Japan Acad. 65 (1989),

5. E. Heinz, Beiträge zur Strüngstheorie der Spektralzerlegung, Math. Ann. 123 (1951), 415-438.

6. E. Kamei, A satellite to Furuta's inequality, Math. Japon 33 (1988), 883-886.

7. T. Kato, Notes on some inequalities for linear operators, Math. Ann. 125 (1952), 208-212.

8. K. Löwner, Über monotone Matrixfunktion, Math. Z. 38 (1934), 177-216.

9. G. K. Pedersen, Some operator monotone functions, Proc. Amer. Math. Soc. 36 (1972), 309-310.

Department of Applied Mathematics, Faculty of Science, Science University of Tokyo, 1-3 Kagurazaka, Shinjuku-ku, Tokyo, 162 Japan 